\title{
Endoscopic orbital decompression for thyroid eye disease
}

\begin{abstract}
Background Thyroid eye disease is a disorder of immune function resulting in extraocular myopathy and an increase in retrobulbar fat. These changes lead to the clinical features found in thyroid eye disease including proptosis, eyelid retraction, restrictive myopathy and sometimes compressive optic neuropathy. Orbital decompression is undertaken for optic nerve compression, exposure keratopathy or cosmesis. Previously this involved an external approach with the removal of the medial orbital wall along with the floor. An intranasal endoscopic approach with the removal of the medial orbital wall and part of the floor has recently been advocated as an alternative procedure. Methods We present the results of 7 patients who underwent endoscopic orbital decompression. The effects on optic nerve function, proptosis and ocular motility were assessed. Symptoms before and after surgery were monitored.

Results The surgery was found to be successful in all cases with a reduction in proptosis and minimal effect on ocular motility. The surgery was also associated with a low post-operative morbidity.

Conclusion We suggest endoscopic orbital decompression is an effective and safe treatment for dysthyroid eye disease.
\end{abstract}

Key words Endoscopic, Dysthyroid eye disease

Thyroid eye disease is an autoimmune disorder in which there is lymphocytic infiltration of the extraocular muscles, and an accumulation of

J.S. Elston

Oxford Eye Hospital

Oxford, UK

B. Koay

G.E.M. Bates

ENT Department

Radcliffe Infirmary

Oxford, UK

Mr R.H.Y. Asaria

Moorfields Eye Hospital

City Road

London EC1V 2PD, UK

Tel: +44 (0)1712533411

Fax: +44 (0)1712534696 glycosaminoglycans in the orbital fat which

leads to an increase in the volume of the orbital tissue. Depending on the severity, this results in orbital congestion, proptosis, lid retraction, restrictive myopathy with diplopia and in the worst cases corneal exposure and compressive optic neuropathy. ${ }^{1}$

The clinical course is progressive over months to years. Some clinical signs persist after resolution due to cicatricial changes.

Treatment of thyroid eye disease consists of lubrication for corneal exposure, systemic immunosuppression, radiation therapy and
RIAZ H.Y. ASARIA, BOON KOAY, JOHN S. ELSTON, GRANT E.M. BATES surgery. Systemic corticosteroids are useful in the acute phase of the disease. However treatment may need to be continued for a long time, and results in side effects. External beam radiation may be employed to limit the amount of proptosis and optic nerve compression.

Orbital decompressive surgery is indicated if the above treatment modalities fail to control compressive optic neuropathy or severe corneal exposure. It is also used to minimise cosmetic deformity due to proptosis.

Surgical decompression involves removing one to four of the bony orbital walls and incising the orbital periostium allowing the swollen orbital contents to prolapse into the adjacent spaces. This can be achieved either externally or using an endoscopic approach.

This paper reports on the results and complications of endoscopic decompression in such patients with thyroid orbitopathy.

\section{Patients and methods}

Seven patients underwent bilateral endoscopic orbital decompressions. There were 3 men and 4 women with a mean age of 37 (range 19 to 62) years. Indications for surgical decompression are listed in Table 1. Prior therapy included systemic immunosuppression and radiotherapy. Some patients went on to have either squint or oculoplastic surgery.

Preoperative assessment included a complete ophthalmological and orthoptic assessment including Hertel exophthalmometry and Hess charts to assess the degree of exophthalmos and oculomotility. Otolaryngologic assessment included nasal endoscopy to identify any sinus or septal pathology. Imaging included either computerised tomography (CT) or magnetic resonance imaging (MRI). All operations were performed under general anaesthesia. All patients were followed at least 12 months postoperatively.

The aim is to remove the entire medial wall and part of the floor of the orbit so that the orbital contents can prolapse into the ethmoidal and maxillary cavities. A pre-operative nasal examination and coronal CT scan of the sinuses are performed to provide accurate details of the 


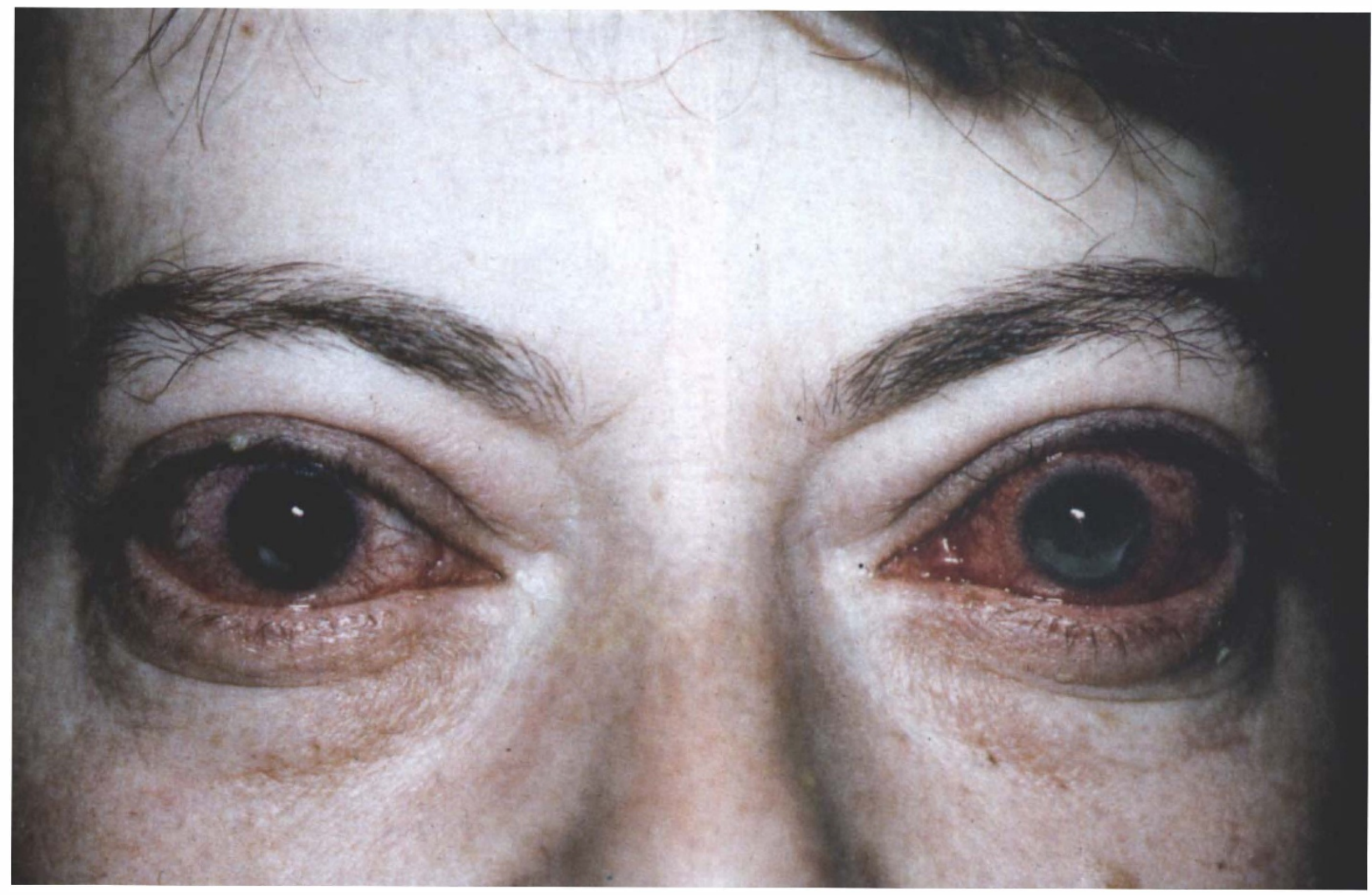

Fig. 1. Preoperative photograph of patient 1 showns severe exophthalmos and exposure keratopathy with bilateral ulceration.

variable ethmoidal anatomy and to identify any coexisting sinonasal pathology.

The operation is performed under general anaesthesia with the patient in a supine position. The nasal cavity is instilled with a mixture of $2 \mathrm{ml}$ of $10 \%$ cocaine and $1 \mathrm{ml}$ mixture of 1:1000 adrenaline solution followed by infiltration of the lateral nasal wall with a $1 \mathrm{ml}$ mixture of 1:80 000 adrenaline and $2 \%$ xylocaine.

A standard endoscopic sphenoethmoidectomy is performed and the medial wall of the orbit is carefully skeletonised and fully exposed as far back as the orbital apex. A generous middle meatal antrostomy is fashioned for access to the orbital floor. The thin bone of the medial orbital wall (lamina papyracea) is removed after careful separation from the periorbita with a Freer elevator.

Bony decompression of the orbital floor extends up to the infraorbital nerve canal to avoid damaging the nerve. Excessive decompression of the orbital floor may also lead to post-operative diplopia or aggravate a preexisting diplopia ${ }^{1}$ and increases the risk of recurrent maxillary sinusitis. ${ }^{2}$ In practice, surgical access to the orbital floor through the middle meatal opening is rather restricted and only the medial 0.5 to $1 \mathrm{~cm}$ of the bone is readily removed. In the presence of thick orbital floor bone, a bone cutting burr may first be used to thin bone down to facilitate removal with a Freer elevator. Care must be taken not to penetrate the periorbita prior to completing all the bone work as premature prolapse of orbital fat may obscure the surgical field.

Once the periorbita is fully exposed, multiple full thickness parallel linear incisions are made with a sickle knife in a posterior-anterior direction. This results in herniation of orbital fat into the ethmoidal and maxillary cavities. Gentle external compression of the globe allows identification of any undivided strands of periorbita which should be completely divided.

If decompression of the optic nerve is required, the thick bone over the orbital apex may first be thinned down with a drill and then removed with a Freer elevator. The aim is to decompress the nerve on its medial half from orbital apex.

Table 1. Patient details

\begin{tabular}{|c|c|c|c|c|c|}
\hline Patient & Age & Sex & Presentation & Surgery & Surgical indications \\
\hline 1 & 33 & $\mathrm{~F}$ & 1995 & 1995 & Exposure keratopathy \\
\hline 2 & 19 & M & 1992 & 1996 & Cosmesis \\
\hline 3 & 32 & M & 1991 & 1995 & Cosmesis \\
\hline 4 & 61 & $\mathrm{~F}$ & 1995 & 1995 & Acute proptosis \\
\hline 5 & 37 & $\mathrm{~F}$ & 1994 & 1995 & Optic neuropathy \\
\hline 6 & 45 & $\mathrm{~F}$ & 1992 & 1996 & Cosmesis \\
\hline 7 & 34 & M & 1992 & 1995 & Cosmesis \\
\hline
\end{tabular}




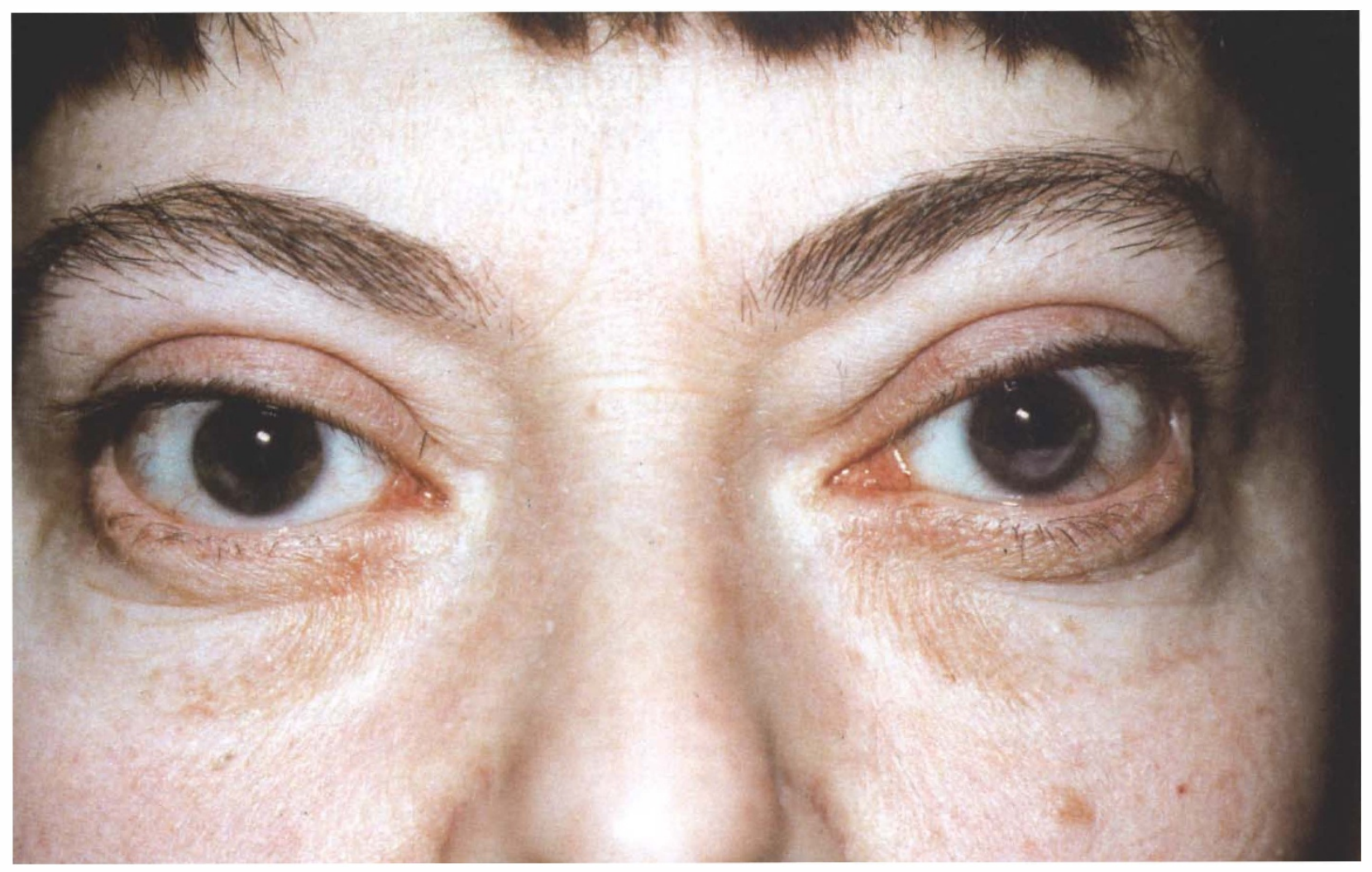

Fig. 2. Post-operative photograph of patient 15 months later following bilateral endoscopic decompressions demonstrates excellent reduction in proptosis with resolution of keratopathy.

The patient is maintained on antibiotics (Augmentin) for 5 days post-operatively and advised not to blow the nose for a week.

\section{Results}

There were no intra-operative or post-operative complications. All the patients except one were discharged within 24 hours. The other patient remained an extra day due to the effect of the general anaesthetic. The follow-up period was at least 12 months. Proptosis was reduced an average of $3.79 \mathrm{~mm}$ (range 2-7). There was no statistical correlation between the reduction in proptosis with age, sex, side of operation, surgical indication, preoperative proptosis, and duration of the disease process.

Four patients whose surgery was performed for cosmesis were pleased with their post-operative appearance. Two of these patients have had oculoplastic surgery. The patient whose surgery was performed for subluxation of the globe and acute proptosis experienced

Table 2. Treatment details of patients

\begin{tabular}{cccc}
\hline Patient & Systemic steroids & Radiotherapy & Other surgery \\
\hline 1 & Yes & No & Oculoplastic \\
2 & Yes & Yes & No \\
3 & Yes & Yes & Oculoplastic \\
4 & No & No & No \\
5 & Yes & Yes & Strabismus \\
6 & Yes & No & No \\
7 & No & No & Strabismus \\
\hline
\end{tabular}

no further episodes. The patient with compressive optic neuropathy had a post-operative improvement in visual acuity with no further loss in optic nerve function. In the seventh patient the exposure keratopathy improved postoperatively.

Two patients had preoperative diplopia, which in one patient increased post-operatively from 18 to 30 prism dioptres base out for near and from 20 to 35 prism dioptres base out for distance. There was minimal postoperative change in the second patient. Both patients went on to have squint surgery. The remaining five patients had no change in oculomotility, with two patients experienced a 'freeing of their eyes'. These results are summarised in Table 3.

\section{Discussion}

Thyroid eye disease can be divided into two broad stages. Initially there is an acute active inflammatory and congestive phase lasting 6-18 months. This stage is mediated by lymphocytes and fibroblasts and is partially responsive to immunosuppression and radiotherapy. ${ }^{1}$ Corticosteroids are the most commonly used immunosuppressive agents, being particularly useful in patients with acute inflammatory orbital disease and optic neuropathy. Although these are improved with steroids, the problem recurs on cessation of therapy. There are many contraindications to, and complications of, steroid therapy. Corticosteroids have little effect on exophthalmos and not all patients respond to treatment. Immunosuppression can also be achieved with 


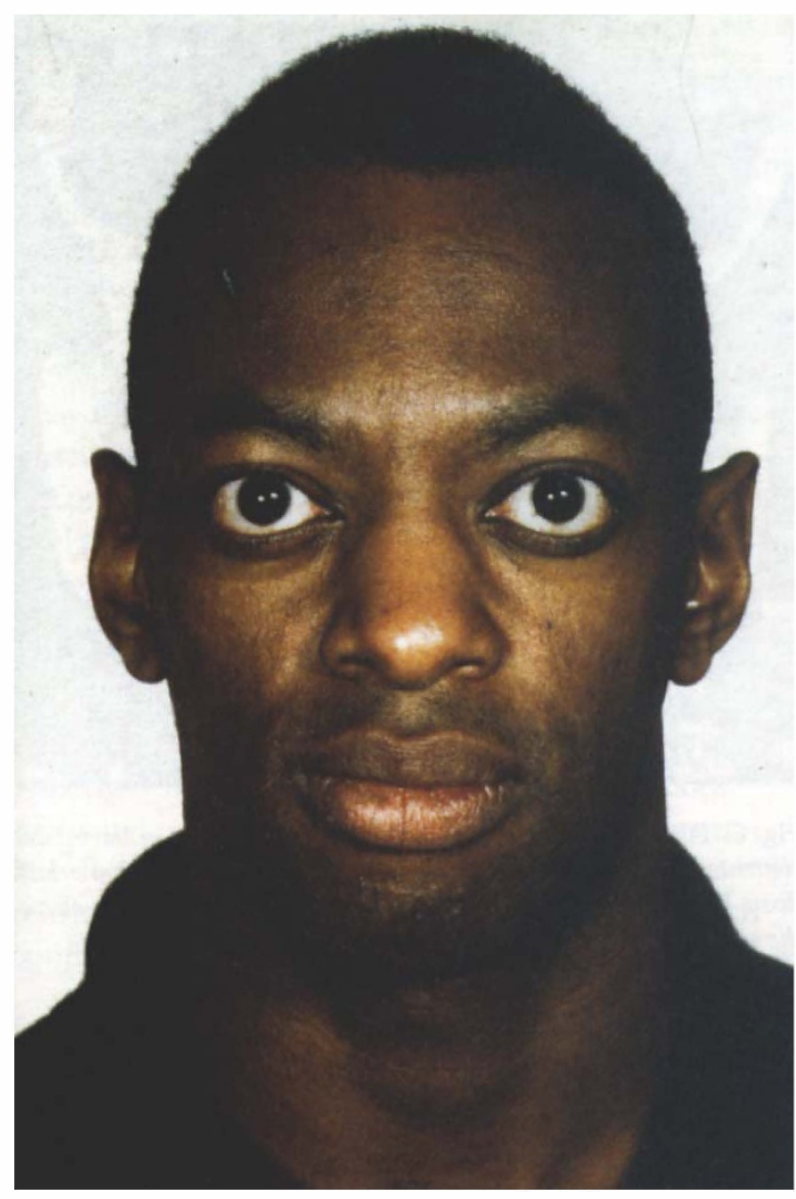

Fig. 3. Preoperative illustration of patient 3 shows marked bilateral exophthalmos.

cyclosporin, methotrexate and azathioprine. All these agents can be associated with serious systemic complications and are not as effective as corticosteroids in controlling the inflammation. ${ }^{1}$

The role of radiotherapy in the treatment of thyroid eye disease is still questioned. A dose of $20 \mathrm{~Gy}$ is recommended by most authors. Radiotherapy is associated with a significant improvement in subjective and objective signs of orbital congestion, in relief of compressive optic neuropathy, and fewer complications than high dose corticosteroids. ${ }^{3}$

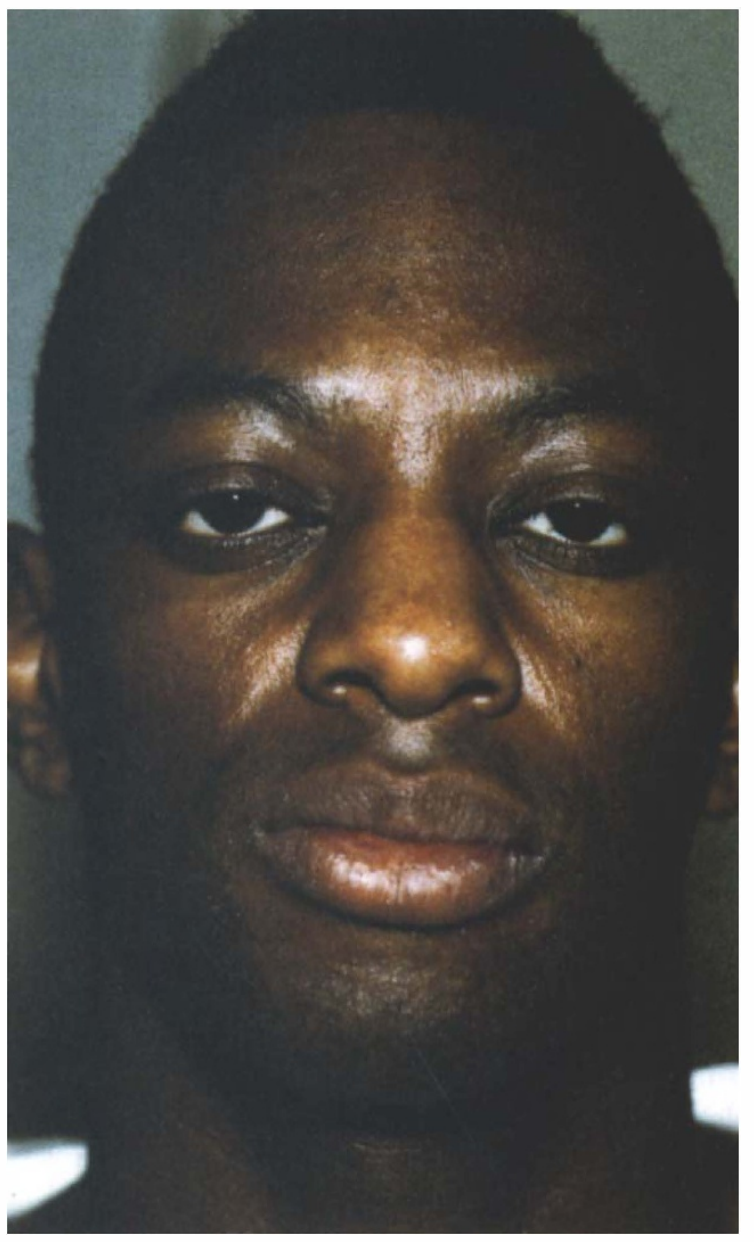

Fig. 4. Post-operative illustration of patient 31 month following bilateral endoscopic decompressions demonstrates an excellent reduction in proptosis with improved cosmesis.

The acute phase is followed by a chronic stable phase with hypertrophy and fibrosis of the extraocular muscles, lacrimal gland and orbital fat. These changes are usually permanent and unresponsive to either steroids or radiotherapy. Surgical treatment is required to treat this stage. ${ }^{4}$ Occasionally, a few patients require orbital decompression in the acute phase when visual acuity is threatened.

Unlike steroids and radiotherapy which try to control the local inflammatory process albeit temporarily, surgery permanently enlarges the orbital volume to

Table 3. A summary of post-operative changes

\begin{tabular}{ccccc}
\hline Name & Surgical indications & Change in proptosis & Change in ocular motility & Change in visual acuity \\
\hline 1 & Exposure keratopathy & $7 \mathrm{~mm}$ & No & Improvement \\
2 & Cosmesis & $2 \mathrm{~mm}$ & No & Nil \\
3 & Cosmesis & $4 \mathrm{~mm}$ & No & Nil \\
4 & Acute proptosis & $4 \mathrm{~mm}$ & No & Nil \\
5 & Optic nerve & $3.5 \mathrm{~mm}$ & No & Improvement \\
6 & Compression cosmesis & $3 \mathrm{~mm}$ & No & Nil \\
7 & Cosmesis & $3 \mathrm{~mm}$ & $18-30 \Delta$ BO (N) & Nil \\
& & & $20-35 \Delta$ BO (D) & \\
\hline
\end{tabular}

$\Delta=$ Prism

$\mathrm{BO}=$ Base out

$(\mathrm{N})=$ Near

(D) $=$ Distance 


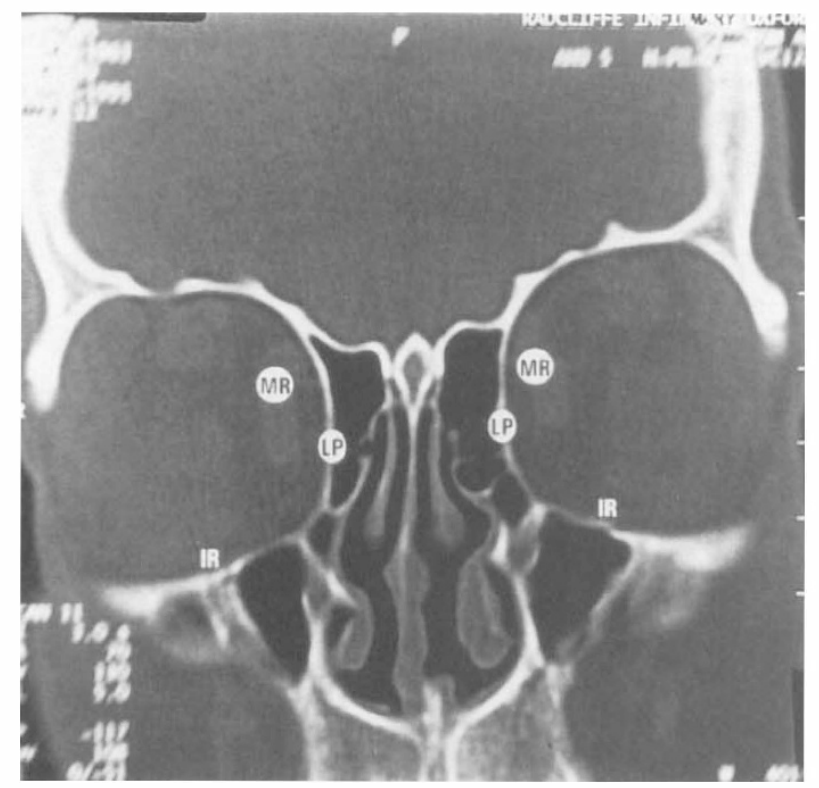

Fig. 5. Preoperative MRI scan. Preoperative coronal view shows congested orbital contents, with an enlarged medial rectus (MR) abutting the lamina papyracea $(L P)$.

reduce the orbital pressure and to improve tissue fluid dynamics. Different surgical approaches have been employed without rigorous comparative evaluation. The timing and indications for surgery in relation to other treatments such as radiotherapy and systemic immunosuppression also require clarification.

Orbital decompression has traditionally been performed through a transantral approach, with the Walsh-Ogura transantral approach being the most commonly used procedure. ${ }^{2}$ A maxillary antrostomy allows removal of the entire orbital floor with the preservation of the intraorbital nerve. The medial orbital wall is exposed and removed through a transantral ethmoidectomy. This approach avoids an external incision, but is associated with the morbidity of a Caldwell-Luc procedure. ${ }^{5}$ The post-operative morbidity includes considerable swelling and discomfort immediately following surgery and persistent numbness over the mandibular region following division of the anterior superior alveolar nerves. The Walsh-Ogura procedure achieves an average of 4 to $6 \mathrm{~mm}$ of reduction in proptosis. A larger reduction in proptosis can be achieved with the Kennerdell-Maroon four wall decompression.

With improved technology in fibreoptic instrumentation orbital structures can be safely and effectively operated on intranasally. Endoscopic dacryocystorhinostomy and orbital decompressions have been shown to be both effective and associated with a low morbidity. ${ }^{6-8}$

Endoscopic orbital surgery allows clear visualisation of orbital structures with no need for a skin incision. Some authors have suggested that severe septal deviation is a contraindication to endoscopic orbital surgery. We do not feel that this is the case as obstructing

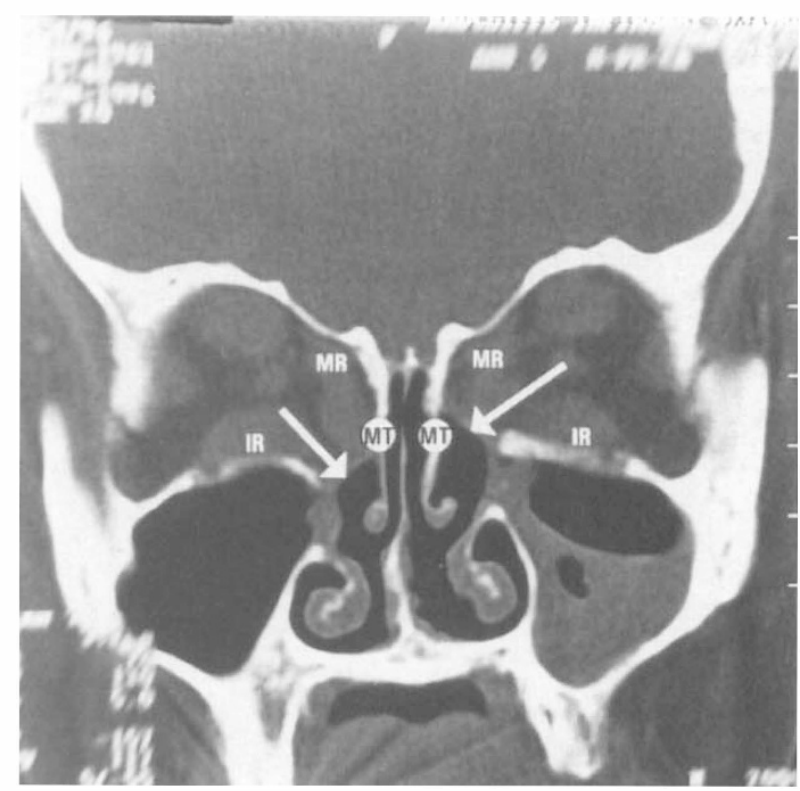

Fig. 6. Post-decompression MRI scan shows expansion of the orbital contents into the ethmoidal and maxillary sinuses. The medial orbital floors have been removed (arrows). The medial rectus now lies above the superior attachments of the middle turbinates (MT).

intranasal pathology is simply treated at the same time. Only 1 of our patients required a minor septoplasty at the time of surgery.

In our series of 14 orbits, we report a reduction in proptosis by an average of $3.79 \mathrm{~mm}$, range 2 to $7 \mathrm{~mm}$. This is in keeping with other reported series of endoscopic decompression. ${ }^{9}$ Kennedy et al. achieved a reduction in proptosis by an average of $4.7 \mathrm{~mm}$, range 3 to $5 \mathrm{~mm}$, in 5 orbits; however two of their procedures involved an exploratory Caldwell-Luc antrotomy with additional decompression of the orbital floor. ${ }^{8}$ Lund et al. achieved a similar reduction in proptosis irrespective of whether an external ethmoidectomy or an endoscopic approach was utilised. ${ }^{10}$ A greater reduction in proptosis can be achieved by a combined external lateral decompression. This technique achieves results comparable to those reported for a conventional WalshOgura procedure while avoiding the morbidity of external ethmoidectomy or Caldwell-Luc antrotomy.

Our results show that endoscopic orbital decompression achieves a similar reduction in proptosis compared with a traditional external approach, and is associated with a lower incidence of post-operative complications. The endoscopic approach avoids postoperative infraorbital nerve anaesthesia as identification of the nerve is possible and it is not traumatised. As visualisation especially in the region of the ethmoid roof and orbital apex is better endoscopically compared with an external approach decompression of the optic nerve is achieved more safely by this approach. Furthermore, an external decompression may be followed by postoperative hypoglobus, ${ }^{11}$ a complication not seen in our patients.

A worsening of extraocular muscle balance may follow orbital decompression with reported incidences 
being as high as $69 \%$ following successful surgery. ${ }^{12}$ McCord has highlighted the relatively low incidence of post-operative diplopia associated with a translid versus the transantral approach. ${ }^{13}$ In his series a $40 \%$ worsening of diplopia was associated with transantral compared with $5.6 \%$ incidence following the translid approach. In our series only 1 patient has a worsening of diplopia after surgery. Furthermore, none of the patients developed a significant A pattern, a complication thought to be caused by disturbance to the origin of the inferior oblique muscle.

Endoscopic decompression can be performed under local anaesthesia. In addition to a more rapid postoperative recovery, the use of local anaesthesia enables intraoperative monitoring of the patient's vision, reducing the likelihood of accidental optic nerve damage.

In conclusion, endoscopic orbital decompression is a safe and effective treatment for dysthyroid eye disease associated with lower post-operative complications compared with conventional external orbital surgery. The decision to enlarge the orbital space should be considered carefully with close co-operation between ophthalmologists and ENT surgeons.

\section{References}

1. Dallow RL, Netland PA. Management of thyroid ophthalmopathy (Graves' disease). In: Albert DM, Jacobiec FA, editors. The principles and practice of ophthalmology. Philadelphia: W.B. Saunders, 1993.
2. McCord DD. Current trends in orbital decompression. Ophthalmology 1985;92:21-33.

3. Shorr N, Neuhaus RW, Baylis HI. Ocular motility problems after orbital decompression for dysthyroid ophthalmology. Ophthalmology 1982;89:323-8.

4. DeSanto LW. The total rehabilitation of Graves' ophthalmology. Laryngoscope 1980;90:1652-78.

5. Walsh TE, Ogura JH. Transantral orbital decompression for malignant exophthalmos. Laryngoscope 1957;67:544-68.

6. Metson R. Endoscopic surgery for lacrimal obstruction. Otolaryngol Head Neck Surg 1991;104:473-9.

7. Kennedy DW. Functional endoscopic sinus surgery technique. Arch Otolaryngol Head Neck Surg 1985;111:643-9.

8. Kennedy DW, Goodstein ML, Miller NR, et al. Endoscopic transantral orbital decompression. Arch Otolaryngol Head Neck Surg 1990;116:275-82.

9. Metson R, Dallow RL, Shore JW. Endoscopic orbital decompression. Laryngoscope 1994;104:950-7.

10. Lund V. Extended applications of endoscopic sinus surgery the territorial imperative. J Laryngol Otol 1997;111:313-5.

11. Long JA, Baylis HI. Hypoglobus following orbital decompression for dysthyroid ophthalmology. Ophthalmic Plast Reconstr Surg 1990;6:185-9.

12. Kazim M, Trokel S, Moore S. Treatment of acute Graves orbitopathy. Ophthalmology 1991;98:1443-8.

13. McCord CD. Orbital decompression for Graves' disease. Exposure through lateral clinical and inferior fornix incision. Ophthalmology 1981;88:533-41. 